Research Article

\title{
Experimental and Numerical Studies on Static Aeroelastic Behaviours of a Forward-Swept Wing Model
}

\author{
Yan Ouyang $\mathbb{D},{ }^{1}$ Kaichun Zeng $\mathbb{D},{ }^{2}$ Xiping Kou $\mathbb{D},{ }^{1}$ Yingsong Gu $\mathbb{D}^{1},{ }^{1}$ and Zhichun Yang $\mathbb{D}^{1}$ \\ ${ }^{1}$ Institute of Structural Dynamics and Control, School of Aeronautics, Northwestern Polytechnical University, \\ $X i$ 'an 710072, China \\ ${ }^{2}$ High Speed Aerodynamics Institute, China Aerodynamics Research and Development Center, Mianyang 621000, China
}

Correspondence should be addressed to Zhichun Yang; yangzc@nwpu.edu.cn

Received 13 January 2021; Accepted 26 May 2021; Published 14 June 2021

Academic Editor: Zeqi Lu

Copyright ( $\odot 2021$ Yan Ouyang et al. This is an open access article distributed under the Creative Commons Attribution License, which permits unrestricted use, distribution, and reproduction in any medium, provided the original work is properly cited.

\begin{abstract}
The static aeroelastic behaviours of a flat-plate forward-swept wing model in the vicinity of static divergence are investigated by numerical simulations and wind tunnel tests. A medium fidelity model based on the vortex lattice method (VLM) and nonlinear structural analysis is proposed to calculate the displacements of the wing structure with large deformation. Follower forces effect and geometric nonlinearity are considered to calculate the deformation of the wing by finite element method (FEM). In the wind tunnel tests, the divergence dynamic pressure is predicted by the Southwell method, and the static aeroelastic displacement is measured by a photogrammetric method. The results obtained by the medium fidelity model calculations show reasonable agreement with wind tunnel test results. A high fidelity model based on coupled computational fluid dynamics (CFD) and computational structural dynamics (CSD) predicts better results of the wing tip displacement when the freestream dynamic pressure is approaching the divergence dynamic pressure.
\end{abstract}

\section{Introduction}

In static aeroelasticity analysis, the interaction between aerodynamics and structural deflections determines the wing bending and twist at every flight condition. The static aeroelastic deformation in the steady flight condition is of great importance because it governs the aerodynamic performance and flight control characteristics [1]. Under the conditions of small structural deformations and small angle of attack (AOA), linear aeroelastic analysis usually gives accurate results. However, if the wing structure undergoes large deformation, the linear calculation may lead to inaccurate predictions. The nonlinear effect usually plays an important role in the structure dynamics $[2,3]$ and may also be crucial in statics. For instance, in the linear aeroelastic calculation, the projection of the deformed wing always coincides with that of the undeformed wing. This indicates a nonphysical effect of lengthening the wing structure without axial load [4]. For the highly flexible joined-wing aircraft, the linear aeroelastic analysis may lead to incorrect prediction of the flight envelope [5].
It is well known that the forward-swept wing and straight wing with high aspect ratio are susceptible to large deformation. When the aerodynamic load is heavy, the deformation of the wing structure will become very large. According to the aerodynamic and structural models used in the nonlinear aeroelastic analysis, numerical models can be categorized into three levels, namely, low, medium, and high fidelity models [6]. Two-dimensional aerodynamic models and nonlinear beam theory are usually used in the low fidelity models [7]. For the high-aspect-ratio wing model, the static and dynamic aeroelastic responses calculated by low fidelity model agree well with the experimental results $[8,9]$. The nonlinear beam theory is capable of calculating the postcritical deformation of a compliant forward-swept wing, and its capability is validated by wind tunnel tests [10].

Though the low fidelity model provides essential insight and knowledge about the aeroelastic characteristics, it has limitation in evaluating the 3D effect of real flow. The strip theory without tip effects correction may overestimate the outboard wing lift and results in greater vertical wing 
displacement and bending rotation [11, 12]. When the spanwise aerodynamic variations are critical for wing structure, the VLM provides a medium fidelity tool for aerodynamic calculation [13]. The VLM can calculate the aerodynamics acting on the wing with large deformation and some pragmatic approaches can be adopted to estimate the effects of drag and camber [14]. The following static structural analysis should be carried out by nonlinear FEM; otherwise, the analysis will yield unrealistic result [15]. Generally speaking, the medium fidelity model is efficient in aeroelastic analysis, so it is widely used in the design optimization process $[16,17]$.

The high fidelity simulation model is based on CFD/CSD coupled method and has been developed rapidly and applied widely in the study of computational aeroelasticity in last decades. Even at low subsonic speeds, much attention should be paid to the effects of structural nonlinearity on the aeroelastic behaviour. The geometric nonlinearity changes the aerodynamic loads, thus leading to considerable errors in the static aeroelastic predictions $[18,19]$. The difference between the maximum deflections of linear and nonlinear wing structure calculations becomes more and more dramatic with increasing aerodynamic loads [20, 21]. Though the low fidelity model gives acceptable results of the static aeroelastic characteristics, the high fidelity model can generate more accurate results and detailed aerodynamic characteristics of the compliant forward-swept wing [22]. The CFD/CSD coupling analysis is essential when the flows are in transonic region because of the shock wave and flow separation $[23,24]$.

The static divergence of wing structure must be predicted accurately by wind tunnel test, because the divergence speed directly reflects the general stiffness of the wing structure and must be considered in the certification process (CS-25 and FAR-25) [1]. There are four static and two dynamic methods used to predict static divergence of wing model in wind tunnel test [25]. All these methods use subcritical response of the experimental model to predict the static divergence. As a static method, the Southwell method is easy to carry out and shows good agreement with the critical divergence test result [26-28]. So, the Southwell method is adopted in the current study.

Before the occurrence of divergence, we are usually concerned about the maximal wing tip displacements. Photogrammetry is a nonintrusive measurement technique commonly used to determine the geometrical information of object by analyzing images recorded by camera. This technique is useful when the object to be measured is inaccessible and noncontact measurement is required, and it is especially suitable for static aeroelastic wind tunnel test. A detailed description of the related theory can be found in [29]. In a series of research, the photogrammetry is proved to be an accurate and powerful method to measure point displacements and local angular deflections [30, 31]. DLTdv7 is a general-purpose digitizing program and it is suitable for two- or three-dimensional video analysis [32]. This program has been widely used in the measurement of wing kinematics [33] and aeroelastic deformation [34]. In the current study, a feasible method based on single camera and DLTdv7 program is used to measure the wing tip displacement in the wind tunnel test.

The study aims to obtain an improved understanding of the static aeroelastic behaviours of a wing model. According to the performance of the available wind tunnel, a forward-swept layout model is used. This layout has a decreased divergence speed compared to the unswept wing due to increased effective AOA. When the free stream dynamic pressure is in the vicinity of the static divergence boundary, the forward-swept wing model will encounter large deformation. The static aeroelastic deformation is calculated by medium and high fidelity models. Wind tunnel tests are performed to validate the simulation results. Finally, we make a comparison between the results of medium and high fidelity models in the aspects of accuracy and efficiency.

\section{Nonlinear Analysis by the Medium Fidelity Model}

An aluminum flat-plate wing model is used in the current study, as shown in Figure 1. The planform of the wing structure is a parallelogram. The semispan and the chord length of the model are $s=750 \mathrm{~mm}$ and $c=200 \mathrm{~mm}$, respectively. The plate thickness is $2.5 \mathrm{~mm}$. The material density is $\rho=2700 \mathrm{~kg} / \mathrm{m}^{3}$. The leading-edge forward-swept angle is $30 \mathrm{deg}$. Three-fifths of the wing root length are fixed. In order to perform nonlinear static analysis efficiently, quadrilateral plate element is used to model the wing structure. Figure 1(a) shows the structure model consisting of $20 \times 60$ elements, whereas Figure 1(b) depicts the corresponding aerodynamic model, which consists of $10 \times 30$ aerodynamic panels.

The equation of motion used for general static aeroelastic calculation can be expressed as follows [35]:

$$
\left(\mathbf{K}_{a a}-q \mathbf{Q}_{a a}\right) \mathbf{u}_{a}+\mathbf{M}_{a a} \ddot{\mathbf{u}}_{a}=q \mathbf{Q}_{a x} \mathbf{u}_{x}+\mathbf{P}_{a},
$$

where $\mathbf{K}_{a a}$ and $\mathbf{M}_{a a}$ are the structural stiffness and mass matrices, respectively. $q$ is the flow dynamic pressure. $\mathbf{u}_{a}$ is the structural displacement vector. $\mathbf{u}_{x}$ is the vector of aerodynamic extra points used to describe the control surface deflections and overall rigid body motions. $\mathbf{Q}_{a a}$ and $\mathbf{Q}_{a x}$ are the aerodynamic influence coefficient matrices corresponding to the structural deformations and unit deflections of the aerodynamic extra points, respectively. $\mathbf{P}_{a}$ is the vector of external loads.

The present wing model has neither control surface nor rigid body motions; equation (1) can be simplified to obtain the divergence dynamic pressure by solving an eigenvalue problem:

$$
\left(\mathbf{K}_{a a}-q \mathbf{Q}_{a a}\right) \mathbf{u}_{a}=0 \text {. }
$$

The eigenvalues $q=q_{d}$ are the dynamic pressures for divergence. Only positive values of $q_{d}$ have physical significance and the lowest value is the critical divergence dynamic pressure.

In static aeroelastic analysis, the downwash can be calculated as [36] 


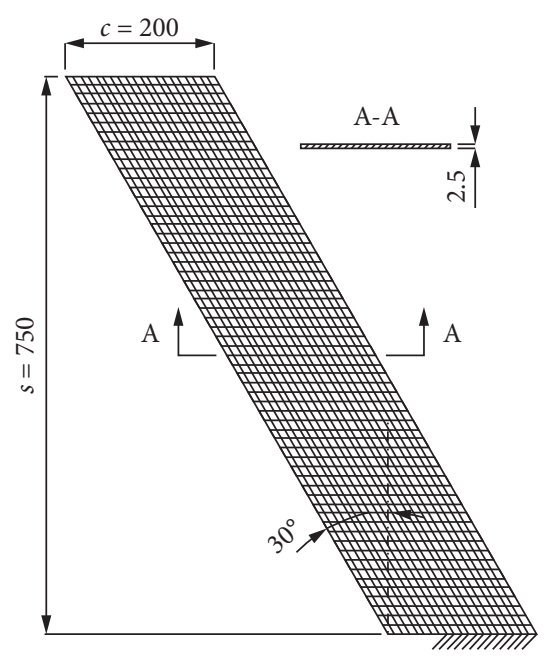

(a)

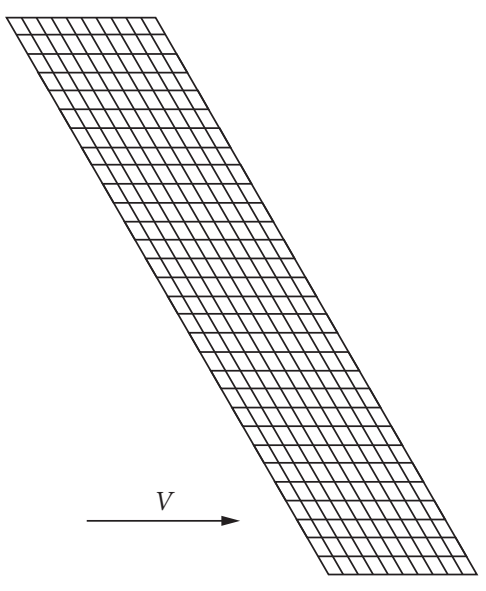

(b)

Figure 1: Forward-swept wing structural and aerodynamic models. (a) Finite element model. (b) Aerodynamic model.

$$
\mathbf{w}_{j}=\mathbf{D}_{j k} \mathbf{u}_{k}+\mathbf{w}_{j}^{g},
$$

where $\mathbf{w}_{j}$ is the downwash vector of the aerodynamic panels and $\mathbf{D}_{j k}$ is the substantial derivative matrix for the aerodynamic displacements. $\mathbf{u}_{k}$ is the vector of aerodynamic displacement and $\mathbf{w}_{j}^{g}$ represents an initial static aerodynamic downwash. The initial AOA, camber, or twist distribution of the wing structure can be taken into account by modifying $\mathbf{w}_{j}^{g}$. Then the theoretical aerodynamic pressures are given by

$$
\mathbf{f}_{j}=q \mathbf{A}_{j j}^{-1} \mathbf{w}_{j},
$$

where $\mathbf{f}_{j}$ is the pressure vector of the aerodynamic panels. $\mathbf{A}_{j j}$ is the aerodynamic influence coefficient matrix, which is a function of Mach number and reduced frequency. The aerodynamic force and moment at aerodynamic grids are obtained by integrating the pressure vector:

$$
\mathbf{P}_{k}=\mathbf{S}_{k j} \mathbf{f}_{j},
$$

where $\mathbf{P}_{k}$ is the vector of force and moment. $\mathbf{S}_{k j}$ is the integration matrix.

To improve the accuracy of aerodynamic calculations, two experimental corrections may be introduced to adjust each theoretical aerodynamic panel lift and moment [37]. These two corrections require that the experimental pressure distribution data at some reference angles of attack be available in advance for numerical calculation. Another correction can be made by adjusting the aerodynamic panels' downwashes [36]. Substitute equations (3) and (4) into (5); the aerodynamic force and moment can be calculated by downwash as

$$
\mathbf{P}_{k}=q \mathbf{S}_{k j} \mathbf{A}_{j j}^{-1}\left(\mathbf{D}_{j k} \mathbf{u}_{k}+\mathbf{w}_{j}^{g}\right) \text {. }
$$

As the downwash of the wing cannot be measured directly in experiments, we use the deformation of the wing model to modify the downwash vector; and an iterative method is used to calculate the static aeroelastic deformation of the wing model.
In static aeroelastic analysis, two types of data transformations are required: the structural equivalent forces from aerodynamic panels to structural grids and the interpolation from the structural deflections to the aerodynamic deflections. The spline methods lead to an interpolation that relates the components of structural grid displacements to the aerodynamic grid displacements. When the deformed structural grids and aerodynamic panels are not coplanar, the local AOA of aerodynamic panel needs to be modified according to structural displacements.

The iteration procedure starts with assigning appropriate initial conditions, as shown in Figure 2. The aerodynamic loads are calculated by VLM. As the aerodynamics is typical follower force, the full geometric nonlinear analysis should be carried out; that is, the aerodynamic loads are treated as following forces and large displacement effect is considered. The surface spline method is applied to transfer the data between structural and aerodynamic grids. The convergence condition of the iteration is that the relative error of the wing tip displacement between two consecutive steps is less than $0.1 \%$. In every iteration cycle, the local AOA, that is the downwash vector of every panel, is updated according to the structural grid displacements. For the purpose of comparison, the results of linear calculation are also included in this paper.

To take the case at AOA $\alpha=1.5 \mathrm{deg}$ and dynamic pressure $q=367 \mathrm{~Pa}$ as an example, the calculation process is shown in Figure 3. The displacements of the leadingedge (LE) point and trailing-edge (TE) point at the wing tip increase and converge gradually after the 22nd iteration step. This indicates that the relative error of the $21 \mathrm{st}$ and $22 \mathrm{nd}$ iteration steps is less than $0.1 \%$. The final displacements of the LE point and TE point at the wing tip are $239.2 \mathrm{~mm}$ and $200.8 \mathrm{~mm}$, respectively. The vertical displacement at the wing tip is more than $30 \%$ of semispan. It means a typical nonlinear case for a flexible wing with large deformation. 


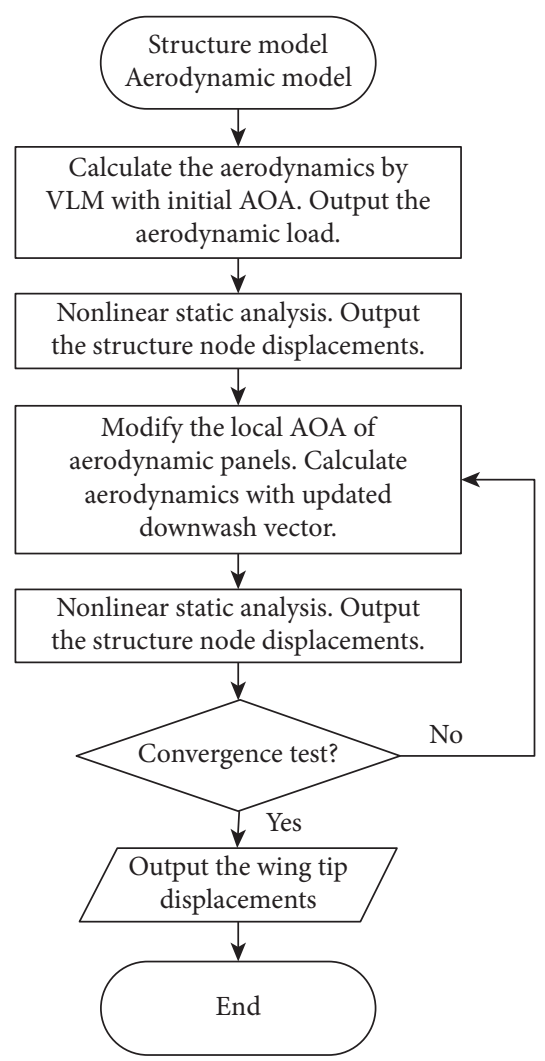

FIGURE 2: Flowchart of nonlinear static aeroelastic analysis.

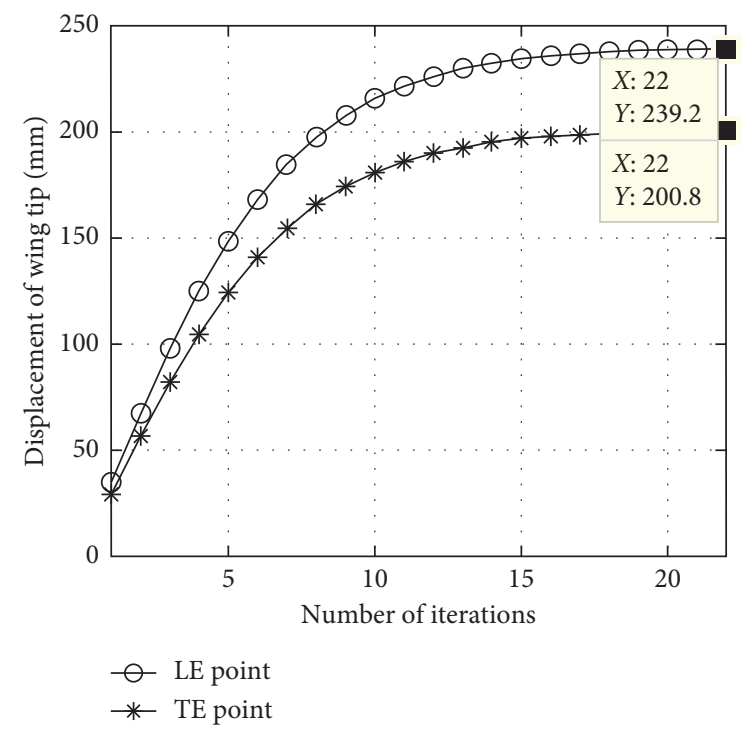

FIgURE 3: Calculation process by medium fidelity model.

\section{Nonlinear Analysis by the High Fidelity Model}

In the detailed design phase of a real wing, a more accurate prediction of static aeroelastic behaviour of the wing structure is necessary. In particular, it is important to predict the static aeroelastic behaviour in the vicinity of divergence with sufficient accuracy. Besides the efficient medium fidelity model, a steady-state CFD/CSD coupling simulation of the wing model is also performed to obtain more accurate results. The simulation is carried out using the commercial software package ANSYS for both the structural analysis and aerodynamic analysis.

The flow field consisting of hexahedral cells around the wing is generated using the ICEM CFD as shown in Figure 4. A common block structure is used, and grid density is varied to obtain three levels of grid, namely, coarse (625000 cells), medium (1036800 cells), and fine levels (1423200 cells). The boundary layer was resolved to make sure that the nondimensional wall distance, $y^{+}$, is less than 1 for all cases. The governing equations used in the ANSYS CFD tool FLUENT are the Reynolds-averaged Navier-Stokes equations, discretized using a conservative and time-implicit colocated finite volume method. The SST $k-\omega$ two-equation turbulence model is used in the density-based solver. The flow density is constant, and velocity inlet boundary condition is used. Diffusion-based smoothing method is adopted to move the wing model boundary in the updating of the fluid volume mesh. This method allows for large boundary deformation and generates good mesh quality. The lift force on the rigid wing is calculated to conduct a grid convergence test. The results of the case at $\alpha=1.5 \mathrm{deg}$ are shown in Figure 5 . The total lifts obtained by medium and fine grids agree very well with the increasing flow dynamic pressure. The results showed a maximal $0.2 \%$ difference between the medium and fine grid solutions. This indicates that the medium level grid is sufficient for analysis. The structural mesh is built in using 2400 3D 20-node solid elements.

\section{Description of Experimental Test Cases}

4.1. Measurement of Wing Tip Displacement. A diagram of the photogrammetric measurement with single camera is shown in Figure 6. The camera is fixed right above the wing tip. Two small pieces of reflective tapes are glued onto the wing surface as optical targets, locating on the LE and TE points at the wing tip. The chord of the undeformed wing tip is on the $x$-axis. Note that the wing tip displacement in the $z$-axis direction, that is, the horizontal displacement, is focused on in this study. In the wind tunnel test, the camera can be fixed outside the flow field, and the aerodynamics on the wing model will not be affected.

During the deformation of the wing, the whole process is recorded by the camera. Later, frames are grabbed from video and used to track the targets. For this purpose, a Sony digital camera with $1920 \times 1080$ pixels is used to acquire images at a rate of 100 frames per second. The measurement error may arise due to nonlinear lens distortion and the inaccurate identification of the target point, so a wind-off experiment is performed to validate this photogrammetric measurement method, as shown in Figure 7. The horizontal displacement of the LE point at the wing tip is set to $105 \mathrm{~mm}$ with static loading. The movement of the LE point is tracked by DLTdv7 and the measured displacement is $106.45 \mathrm{~mm}$. By comparing the measured data with the known displacement of the LE point, we find that the measuring error is less than $1.5 \%$. This indicates that the measurement is accurate enough in assessing the numerical results. 


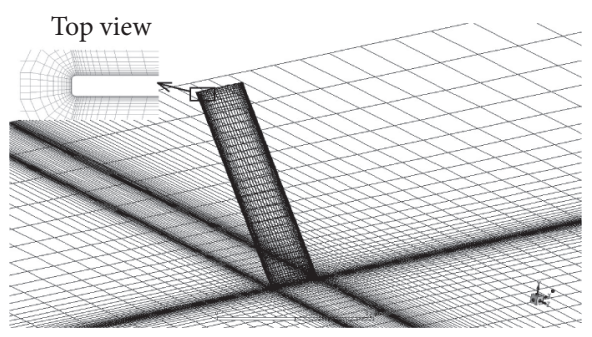

FIgURE 4: Fluid mesh of the CFD/CSD coupling simulation.

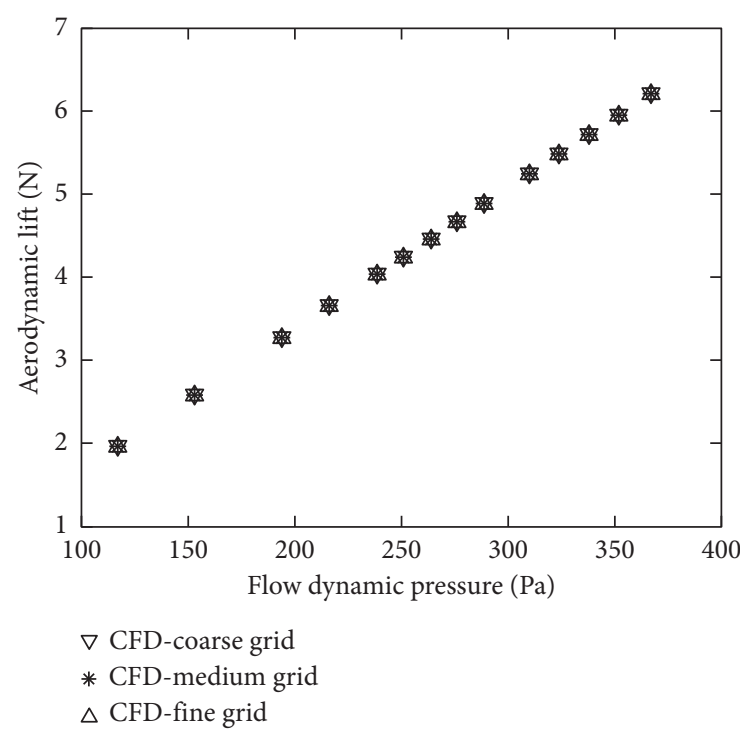

FIgURE 5: Total lift on the rigid wing at $\alpha=1.5 \mathrm{deg}$.
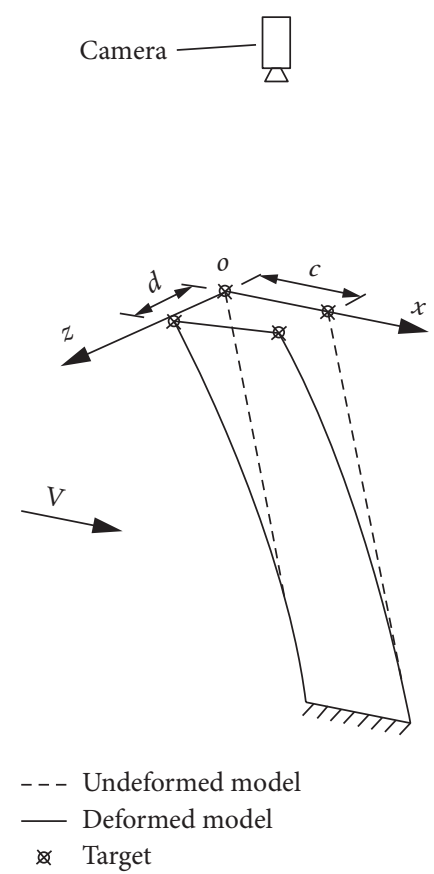

FIgURE 6: Schema of single-camera measurement system.

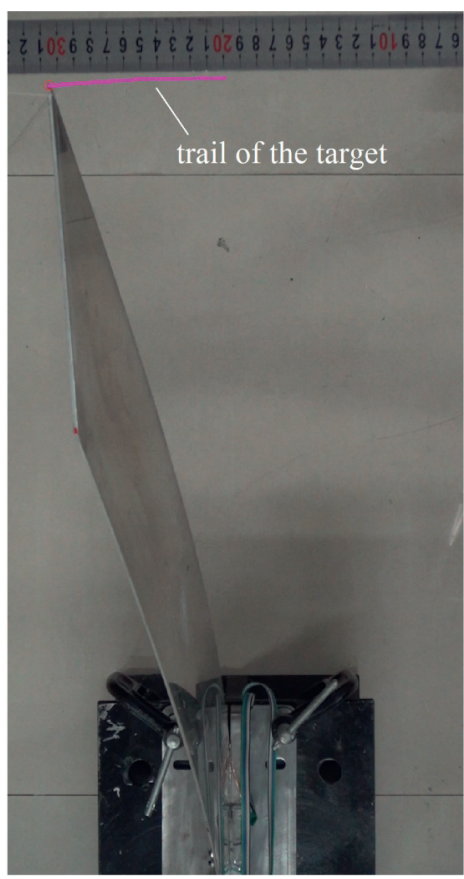

FIgURE 7: Target tracking in image plane for the wind-off experiment.

4.2. Ground Vibration Test. Before conducting the wind tunnel test, it is necessary to perform GVT for model validation and updating. As shown in Figure 8, a SINOCERA shaker (JZK-5) and a power amplifier (YE5874A) are used to excite the model. As the wing structure is very flexible, the drive point is placed near the root of the wing. LMS vibration test system is used to provide random signal to the shaker with a frequency band of 0 to $100 \mathrm{~Hz}$. The force at the joint between the shaker and the model is measured by a PCB force transducer (208C02). The displacement response signal is measured by KEYENCE laser (LK-G150). During the test, the wing structure is kept in microvibration so that the linear case is satisfied. The experimental results of the first five modal shapes and natural frequencies are listed in the first and second column of Table 1, respectively.

After the GVT, a model updating procedure is applied to match the dynamic characteristics of the numerical and the experimental models. The updating procedure performed by solving the optimization problem is defined as follows:

$$
\begin{array}{ll}
\text { Min: } & \sum_{i=1}^{5}\left|\frac{f_{i}^{e}-f_{i}^{s}}{f_{i}^{e}}\right| \\
\text { s.t. } & \left\{\begin{array}{l}
0.5 E_{0} \leq E \leq 1.5 E_{0} \\
0.5 \mu_{0} \leq \mu \leq 1.5 \mu_{0},
\end{array}\right.
\end{array}
$$

where the objective function is the sum of relative errors of the first five natural frequencies obtained by the experiment and simulation. The design variables are Young's modulus $E$ and Poisson's ratio $\mu$ of the finite element model. $E_{0}=71 \mathrm{GPa}$ and $\mu_{0}=0.33$ are the standard material properties of aluminum alloy. Optimization problem 


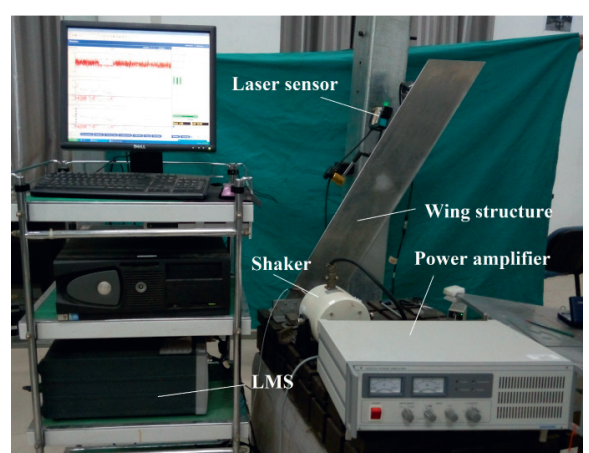

FIGURE 8: Ground vibration test of the forward-swept wing.

TABLE 1: The first five natural frequencies and modal shapes of the wing model.

\begin{tabular}{lccc}
\hline Order (mode) & GVT (Hz) & $\begin{array}{c}\text { Calculated by } \\
\text { FEM (Hz) }\end{array}$ & $\begin{array}{c}\text { Relative } \\
\text { error (\%) }\end{array}$ \\
\hline 1st (1B) & 2.39 & 2.41 & 0.84 \\
2nd (2B) & 15.45 & 15.16 & 1.88 \\
3rd (1T) & 24.15 & 24.07 & 0.33 \\
4th (3B) & 43.93 & 42.87 & 2.41 \\
5th (2T) & 72.46 & 72.61 & 0.21 \\
\hline
\end{tabular}

equation (7) is solved by Optimization Toolbox in MATLAB, and the final solutions are $E=60.37 \mathrm{GPa}$ and $\mu=0.31$. The natural frequencies of two models are listed in Table 1. For each of the first five natural frequencies, the relative errors between the computational and experimental values are very small, so the finite element models are validated.

4.3. Wind Tunnel Test. The forward-swept wing model is tested in the NWPU NF-2 acoustic wind tunnel, which is an opening circuit tunnel with a test section of $\Phi 1.5 \mathrm{~m}$ and a length of $2 \mathrm{~m}$. The wing model is mounted vertically on a turntable that can rotate to change AOA of the wing, as shown in Figure 9. A wire and pulley mechanism is used to protect the wing model. The wires are loose during the experiment and can be tightened to pull the wing tip from both sides in case of excessive large deformation of the wing model. The experiment process is recorded by the digital camera from the top view of the wind tunnel.

The spanwise bending strain is used in the prediction of the static divergence. For this purpose, two strain gages are used. The lower gage was located at the root of the wing model and the upper one at the fifth semispan station $((y / s)=20 \%)$. The strain response signal is measured by a dynamic strain data acquisition system. At the beginning of the experiment, the turntable is adjusted to ensure that the initial AOA of the wing is $0 \mathrm{deg}$. Three cases, namely, $\alpha=0.5 \mathrm{deg}, 1.0 \mathrm{deg}$, and $1.5 \mathrm{deg}$, are used to follow the Southwell method.

The divergence dynamic pressure results predicted by experiment and simulation are listed in Table 2. The results obtained by the two strain gages show good agreement, because the Southwell method is independent of the strain gage location. The AOA condition affects the prediction of divergence dynamic pressure and smaller AOA generates higher dynamic pressure prediction. Equation (2) yields a prediction of $q_{d}^{s}=382.5 \mathrm{~Pa}$, which agrees well with the experiment result $q_{d}^{e}=377.3 \mathrm{~Pa}$ when $\alpha=0.5 \mathrm{deg}$. Finally, at every case of AOA, the average of the two predicted divergence dynamic pressures is treated as the experiment result.

A typical deformation process of wing model in the wind tunnel tests is shown in Figure 10. The movements of LE and TE points are tracked by DLTdv7 continuously. Under this experimental condition, the final measured displacements of the LE point and TE point at the wing tip are $163.0 \mathrm{~mm}$ and $140.2 \mathrm{~mm}$, respectively.

\section{Results and Discussion}

Static aeroelastic deformations of the wing model are computed at two angles of attack ( $\alpha=1.0 \mathrm{deg}$ and $1.5 \mathrm{deg})$. The three simulation approaches are VLM coupled with linear structure assumptions, the VLM coupled with the nonlinear structural solver described in the second section, and the CFD/CSD coupling method. The results of three numerical approaches are compared with the experiment data. In order to protect the test model from damage, the performed tests are limited to the subcritical states. The maximum dynamic pressures of the flow are $310 \mathrm{~Pa}$ and $289 \mathrm{~Pa}$ for the cases of $\alpha=1.0 \mathrm{deg}$ and $1.5 \mathrm{deg}$, respectively; and, in the simulations, the maximum dynamic pressure is set up to a much higher level. The difference among the three simulation results will be shown further.

For $\alpha=1.0 \mathrm{deg}$, the results are shown in Figure 11. When the dynamic pressure is low, the wing tip displacements increase gradually with increasing dynamic pressure. When the dynamic pressure is above the experimental predicted divergence boundary $q_{d}^{e}$, the wing tip displacements increase dramatically. Under the low dynamic pressure condition (lower than $275 \mathrm{~Pa}$ ), the deformation of the wing model is small, and the geometric nonlinearity is not reflected, so the results of two medium fidelity models and experiment results agree well. When the dynamic pressure is above $287 \mathrm{~Pa}$, the differences between the simulation and experiment results are obvious. The medium fidelity model results are always smaller than those of experiments, while the high fidelity model results are always slightly larger. The effect of the geometric nonlinearity may strengthen the structure, so the wing tip displacement will not tend to infinity when the geometric nonlinearity is taken into account.

Figure 12 shows the results of the case at $\alpha=1.5 \mathrm{deg}$. The same as the aforementioned case, the difference between the results of two medium fidelity models and experiment becomes more and more significant with increasing dynamic pressure. In contrast, the CFD/CSD results agree very well with experiment results, with a slight overestimation of the displacements. When the dynamic pressure is higher than $q_{d}^{e}=357.5 \mathrm{~Pa}$, the results obtained by linear calculation are totally unrealistic, because the wing tip displacements are even larger than the semispan. Thus, the medium fidelity model based on linear method has limitations when the flow 


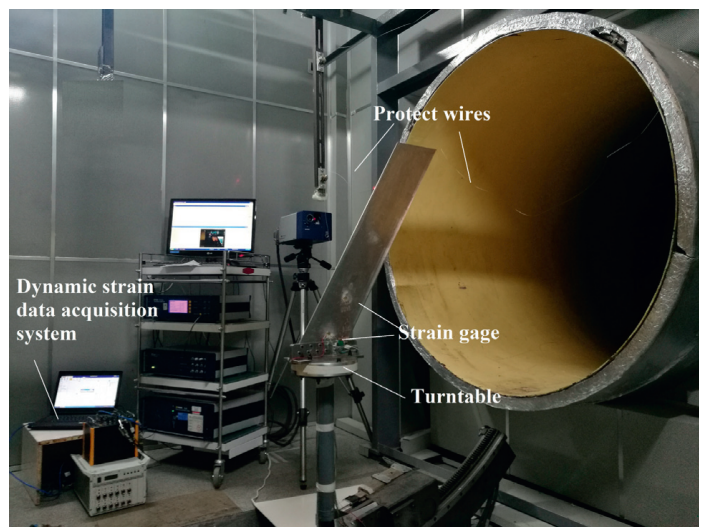

FIGURE 9: Forward-swept wing model in the test section of wind tunnel (upstream view).

TABLE 2: Divergence dynamic pressure predicted by wind tunnel test and simulation.

\begin{tabular}{lcccc}
\hline & \multicolumn{2}{c}{$q_{d}^{e}$ predicted by Southwell method (Pa) } & \multirow{2}{*}{$q_{d}^{s}$ calculated by equation (2) (Pa) } \\
\hline By upper strain gage & $\alpha=0.5 \mathrm{deg}$ & $\alpha=1.0 \mathrm{deg}$ & $\alpha=1.5 \mathrm{deg}$ & 382.5 \\
By lower strain gage & 371.6 & 355.1 & 338.1 & 337.7 \\
Average & 383.0 & 359.5 & 337.9 & \\
\hline
\end{tabular}

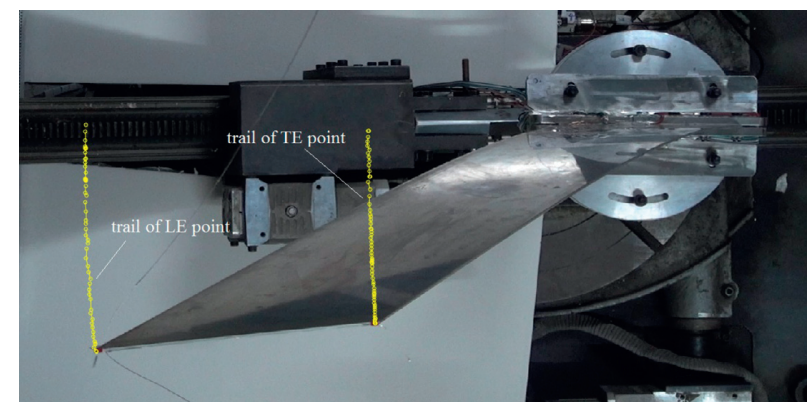

Figure 10: Wing deformation process at $\alpha=1.5 \mathrm{deg}$, from $q=0$ to $287 \mathrm{~Pa}$.

dynamic pressure approaches the divergence dynamic pressure. The medium fidelity model based on nonlinear method may always underestimate the deformation but keep physical meaning.

The data of the curves in Figures 11 and 12 are listed in Tables 3 and 4, respectively. When the AOA is $\alpha=1.0 \mathrm{deg}$, the accuracies of the medium fidelity and high fidelity models are almost the same. For example, when the dynamic pressure is $310 \mathrm{~Pa}$, the relative errors of the VLM + linear, $\mathrm{VLM}+$ nonlinear, and CFD/CSD are 19\%, 26\%, and $27 \%$, respectively. The data in Table 4 show that the CFD/CSD has better accuracy when $\alpha=1.5 \mathrm{deg}$. For example, when the dynamic pressure is $289 \mathrm{~Pa}$, the relative errors of the $\mathrm{VLM}+$ linear, $\mathrm{VLM}+$ nonlinear, and CFD/CSD are $31.15 \%$, $36.96 \%$, and $2.33 \%$, respectively. This indicates that when the deformation is large, a high fidelity model is required in the aerodynamic calculation.

In order to find the primary factor for the difference among the three numerical approaches, we compare the status of deformed structure at the beginning of the iterations. Here, we only focus on the case of $\alpha=1.5 \mathrm{deg}$ and $q=367 \mathrm{~Pa}$ for brevity. The deformation of the wing structure is calculated under the aerodynamics of rigid wing, and the results are listed in Table 5. As the wing tip displacements are almost the same, this indicates that the three approaches generate similar results for their following iteration process; and the discrepancy of the final structure deformation mainly comes from the iteration process.

When the iteration process is finished, the wing structure is in a static state under the balance of the aerodynamic load and structure elastic restoring forces. It is well reflected from Figure 13, where the streamlines of the deformed wing at different dynamic pressure are plotted. These CFD calculation results show the flow field near the wing model. When the dynamic pressure is $q=216 \mathrm{~Pa}$, as shown in Figure 13(a), the deformation of the wing model is small. The wingtip vortex appears because of the three-dimensional effect. Flows near the wing root and midspan are completely 

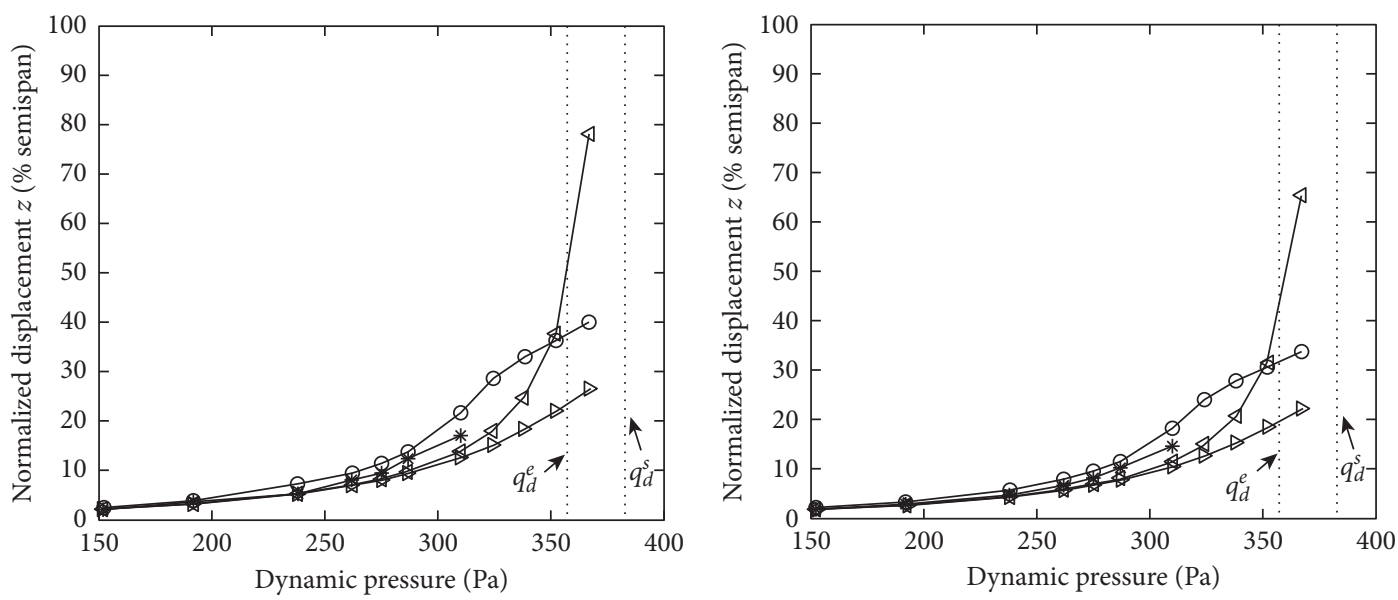

\footnotetext{
$\triangleleft$ LE-medium fidelity model (linear)

$\rightarrow$ LE-medium fidelity model (nonlinear)

* LE-experiment

$\multimap$ LE-high fidelity model (CFD/CSD)
}

(a)

$$
\begin{aligned}
& \triangleleft \text { TE-medium fidelity model (linear) } \\
& \rightarrow \quad \text { TE-medium fidelity model (nonlinear) } \\
& * \quad \text { TE-experiment } \\
& \multimap \quad \text { TE-high fidelity model (CFD/CSD) }
\end{aligned}
$$

(b)

Figure 11: Wing tip displacements versus dynamic pressure for $\alpha=1.0 \mathrm{deg}$. (a) LE point. (b) TE point.

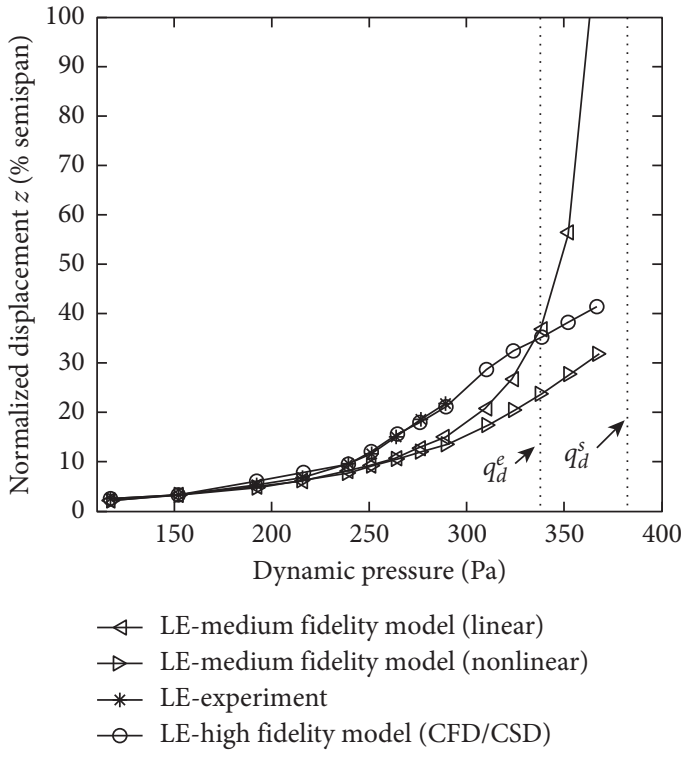

(a)

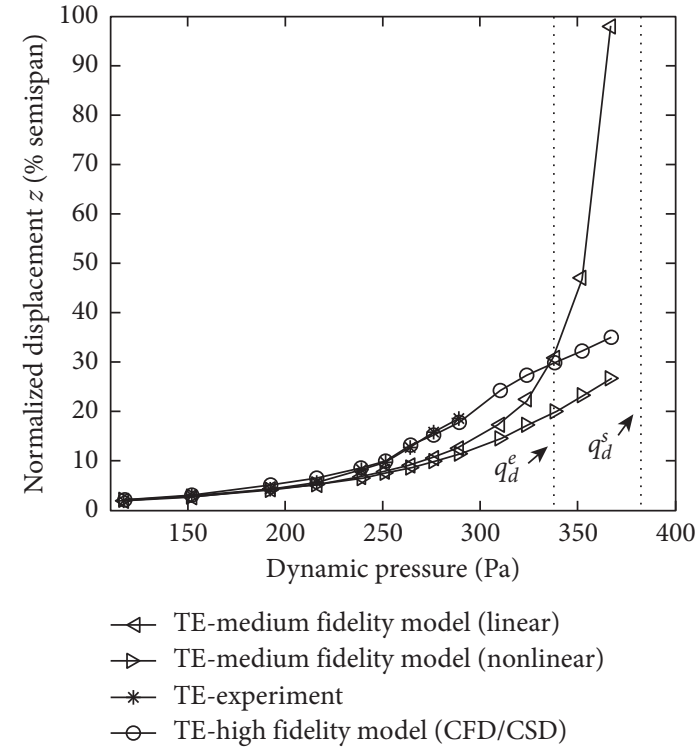

(b)

Figure 12: Wing tip displacements versus dynamic pressure for $\alpha=1.5 \mathrm{deg}$. (a) LE point. (b) TE point.

attached. Figure $13(\mathrm{~b})$ is the case of $q=264 \mathrm{~Pa}$; flow moves toward the wing root obviously because of the forwardswept effect. Flow separation occurs firstly at the wing root. When the dynamic pressure increases to $q=310 \mathrm{~Pa}$, the nondimensional wing tip displacement is larger than $20 \%$, and the local AOA at the wing root and midspan are $8.5 \mathrm{deg}$ and $9.6 \mathrm{deg}$, respectively. As shown in Figure 13(c), flow separation has extended to the midspan station. Figure 13(d) shows the case of $q=367 \mathrm{~Pa}$, and vortices at the wing tip and midspan become stronger than ever. The flow field near the wing model is very complex and fully separated. The VLM is not suitable for this case, and the calculation accuracy cannot be guaranteed. Thus, we can see that the CFD/CSD method has the highest accuracy.

Further insights can be gained by checking the pressure difference distribution when the iteration is finished. A comparison between the pressure difference distributions provided by the medium and high fidelity models is shown in Figure 14 at five spanwise locations $(y / s)=1.67 \%, 25 \%, 48.33 \%, 75 \%$, and $98.33 \%$. Station 1 is 
TABLE 3: Wing tip displacements when $\alpha=1.0 \mathrm{deg}$, \%semispan.

\begin{tabular}{|c|c|c|c|c|c|c|c|c|}
\hline \multirow{2}{*}{$q(\mathrm{~Pa})$} & \multicolumn{4}{|c|}{ LE } & \multicolumn{4}{|c|}{$\mathrm{TE}$} \\
\hline & VLM + linear & VLM + nonlinear & Experiment & $\mathrm{CFD} / \mathrm{CSD}$ & VLM + linear & VLM + nonlinear & Experiment & $\mathrm{CFD} / \mathrm{CSD}$ \\
\hline 152 & 2.12 & 2.13 & 2.11 & 2.59 & 1.78 & 1.78 & 1.85 & 2.17 \\
\hline 192 & 3.25 & 3.25 & 3.56 & 4.08 & 2.72 & 2.72 & 3.04 & 3.42 \\
\hline 238 & 5.31 & 5.29 & 5.64 & 7.00 & 4.45 & 4.43 & 4.85 & 5.86 \\
\hline 262 & 7.01 & 6.92 & 8.08 & 9.57 & 5.87 & 5.80 & 6.67 & 8.02 \\
\hline 275 & 8.26 & 8.07 & 9.43 & 11.48 & 6.91 & 6.76 & 8.09 & 9.63 \\
\hline 287 & 9.71 & 9.34 & 12.40 & 13.78 & 8.13 & 7.83 & 10.42 & 11.56 \\
\hline 310 & 13.83 & 12.58 & 17.09 & 21.65 & 11.57 & 10.53 & 14.57 & 18.22 \\
\hline 324 & 17.93 & 15.16 & & 28.55 & 15.01 & 12.71 & & 24.07 \\
\hline 338 & 24.65 & 18.32 & & 33.09 & 20.63 & 15.36 & & 27.92 \\
\hline 352 & 37.62 & 22.02 & & 36.40 & 31.49 & 18.46 & & 30.73 \\
\hline 367 & 78.19 & 26.47 & & 40.00 & 65.44 & 22.21 & & 33.78 \\
\hline
\end{tabular}

TABLe 4: Wing tip displacements when $\alpha=1.5 \mathrm{deg}$, \%semispan.

\begin{tabular}{|c|c|c|c|c|c|c|c|c|}
\hline \multirow{2}{*}{$q(\mathrm{~Pa})$} & \multicolumn{4}{|c|}{ LE } & \multicolumn{4}{|c|}{$\mathrm{TE}$} \\
\hline & VLM + linear & VLM + nonlinear & Experiment & $\mathrm{CFD} / \mathrm{CSD}$ & VLM + linear & VLM + nonlinear & Experiment & $\mathrm{CFD} / \mathrm{CSD}$ \\
\hline 117 & 2.13 & 2.13 & 2.57 & 2.51 & 1.78 & 1.78 & 1.90 & 2.11 \\
\hline 153 & 3.22 & 3.22 & 3.42 & 3.88 & 2.70 & 2.70 & 2.92 & 3.25 \\
\hline 194 & 4.97 & 4.96 & 5.44 & 6.16 & 4.16 & 4.16 & 4.50 & 5.17 \\
\hline 216 & 6.27 & 6.23 & 6.77 & 7.90 & 5.25 & 5.22 & 5.69 & 6.62 \\
\hline 239 & 8.06 & 7.92 & 9.62 & 10.33 & 6.75 & 6.64 & 8.25 & 8.66 \\
\hline 251 & 9.24 & 9.01 & 11.57 & 12.01 & 7.73 & 7.54 & 9.76 & 10.07 \\
\hline 264 & 10.78 & 10.37 & 15.05 & 15.72 & 9.03 & 8.69 & 12.71 & 13.19 \\
\hline 276 & 12.55 & 11.85 & 18.69 & 16.75 & 10.51 & 9.92 & 15.81 & 14.07 \\
\hline 289 & 14.98 & 13.71 & 21.75 & 21.25 & 12.54 & 11.49 & 18.62 & 17.89 \\
\hline 310 & 20.74 & 17.42 & & 28.81 & 17.37 & 14.60 & & 24.30 \\
\hline 324 & 26.91 & 20.42 & & 32.50 & 22.52 & 17.12 & & 27.42 \\
\hline 338 & 36.98 & 23.84 & & 35.47 & 30.96 & 19.99 & & 29.93 \\
\hline 352 & 56.44 & 27.62 & & 38.29 & 47.24 & 23.17 & & 32.34 \\
\hline 367 & 117.30 & 31.89 & & 41.51 & 98.18 & 26.77 & & 35.07 \\
\hline
\end{tabular}

TABle 5: The wing tip displacements when applying the aerodynamics of rigid wing $(\mathrm{mm})$.

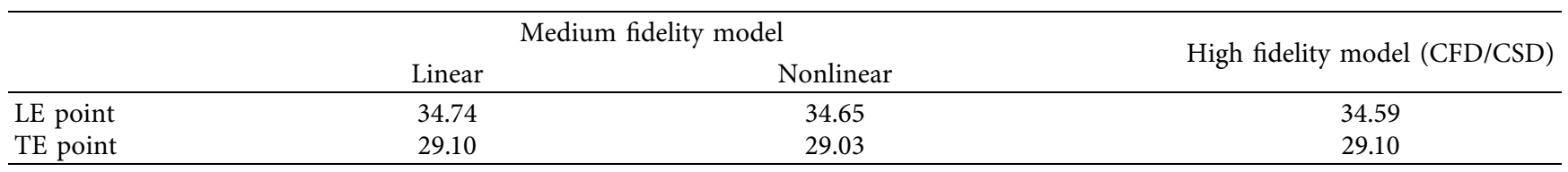

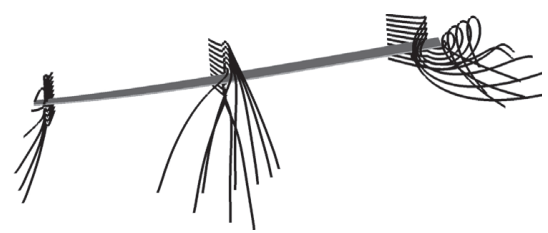

(a)

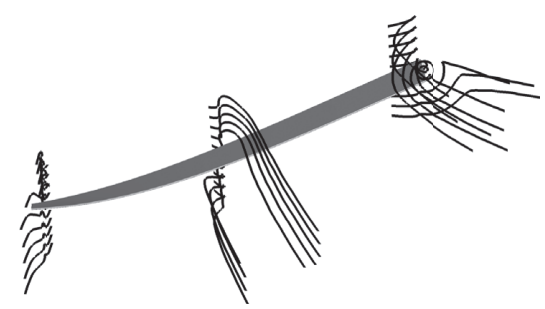

(c)

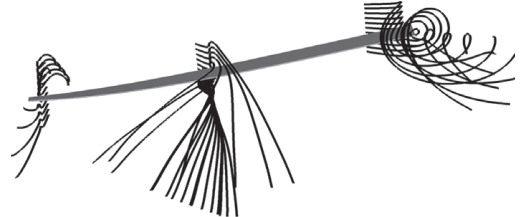

(b)

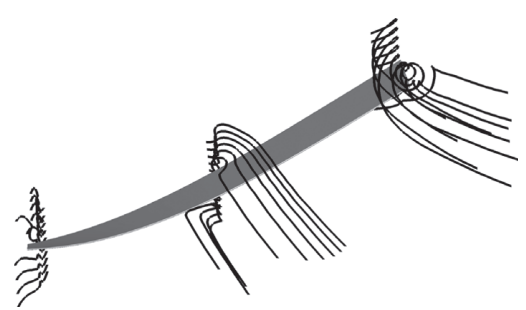

(d)

FIGURE 13: Streamlines of deformed wing at different dynamic pressure, $\alpha=1.5$ deg. (a) $q=216 \mathrm{~Pa}$. (b) $q=264 \mathrm{~Pa}$. (c) $q=310 \mathrm{~Pa}$. (d) $q=367 \mathrm{~Pa}$. 


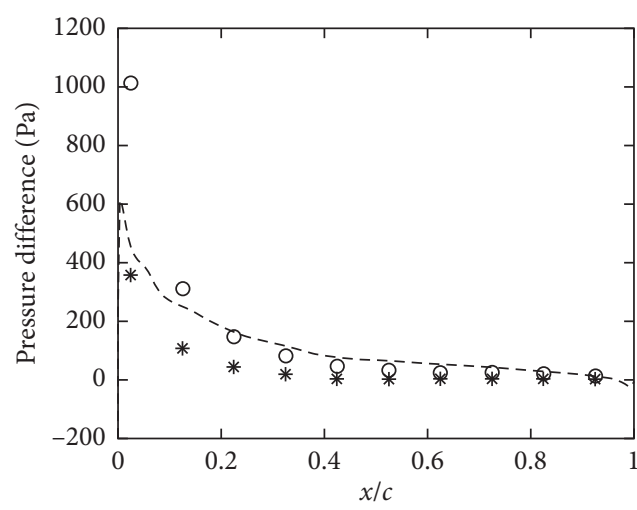

○ Medium fidelity model (linear)

* Medium fidelity model (nonlinear) - - - High fidelity model (CFD/CSD)

(a)

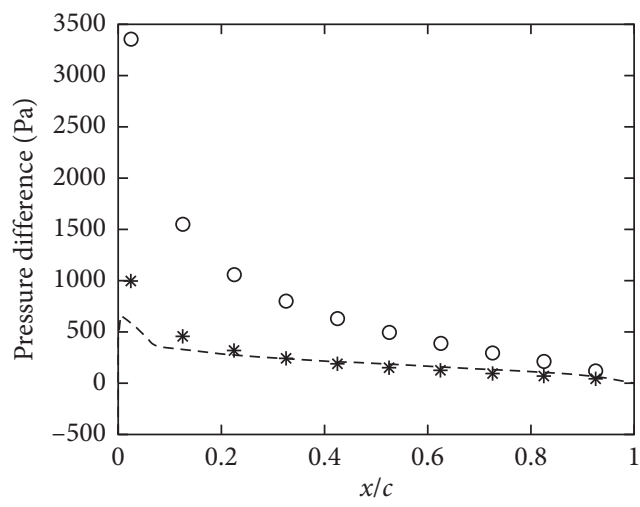

○ Medium fidelity model (linear)

* Medium fidelity model (nonlinear) - - - High fidelity model (CFD/CSD)

(c)

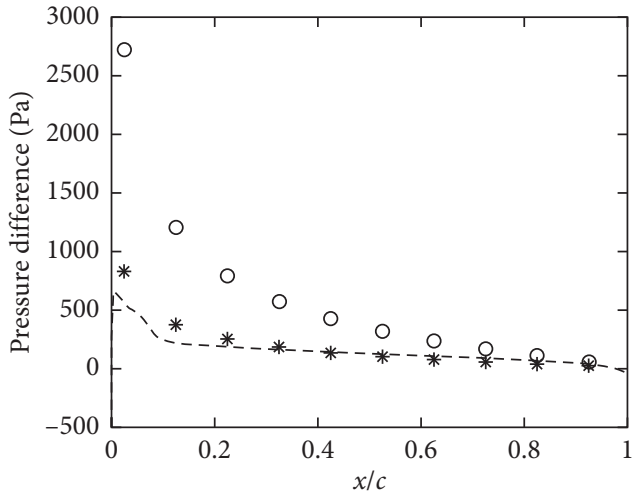

○ Medium fidelity model (linear)

* Medium fidelity model (nonlinear)

- - High fidelity model (CFD/CSD)

(b)

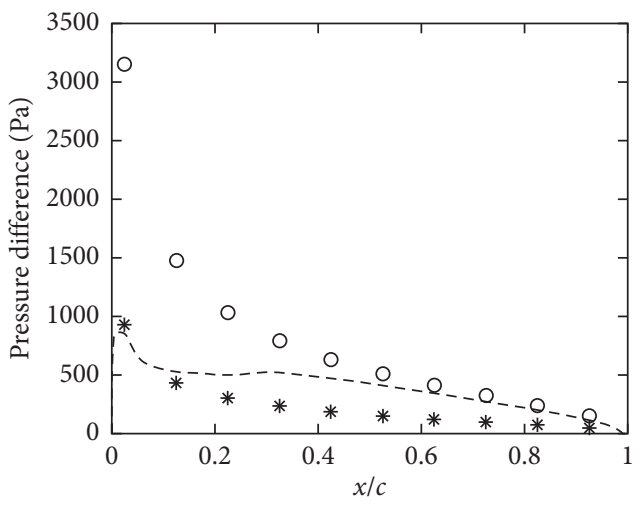

○ Medium fidelity model (linear)

* Medium fidelity model (nonlinear)

- - - High fidelity model (CFD/CSD)

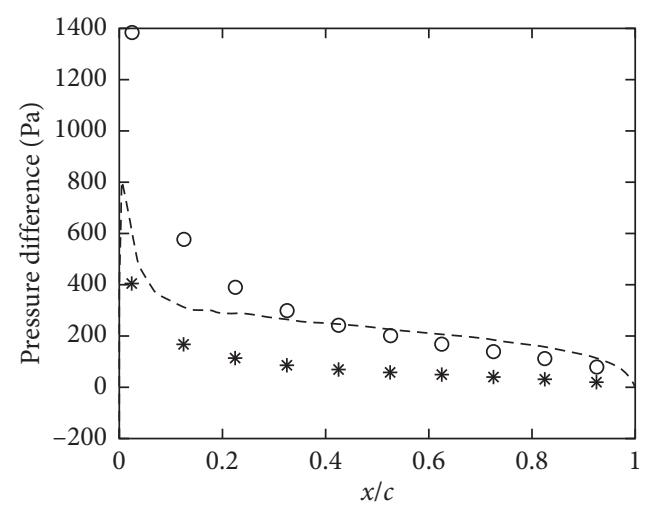

○ Medium fidelity model (linear)

* Medium fidelity model (nonlinear)

- - - High fidelity model (CFD/CSD)

(e)

Figure 14: Pressure difference distribution at $\alpha=1.5 \mathrm{deg}, q=367 \mathrm{~Pa}$. (a) Station $1((y / s)=1.67 \%)$. (b) Station $2((y / s)=25 \%)$. (c) Station $3((y / s)=48.33 \%)$. (d) Station $4((y / s)=75 \%)$. (e) Station $5((y / s)=98.33 \%)$. 
close to the fixed wing root, so the pressure difference distribution obtained by three methods is close except the region near the LE. At stations 2 and 3, the chordwise pressure evolutions calculated by nonlinear method and CFD/CSD coupling are close; and the linear method generates much larger values. In the outboard wing $((y / s)=75 \%)$ and the wing tip $((y / s)=98.33 \%)$, pressure difference distribution calculated by CFD/CSD coupling method becomes closer to the distribution obtained by linear method. When using the nonlinear method, the underestimation of the lift in the outboard wing leads to the underestimation of the structure deformation.

It is worth mentioning here that, with the same computation resource (CPU: Intel Xeon E5-26202.10 GHz, 32 cores), the average computation time of CFD/CSD coupling for a single case is more than 20 hours, while the presented nonlinear analysis on the basis of medium fidelity model takes less than 10 minutes. This difference is mainly caused by the aerodynamics calculation, because the CFD method (solving the NS equations) is much more time-consuming than the VLM. A compromise between the result accuracy and the computation efficiency should be made. When the structure deformation is small, the linear and nonlinear methods based on medium fidelity model have great advantage in efficiency with acceptable accuracy. If the priority is accuracy, the CFD/CSD coupling method can be used especially when the freestream dynamic pressure is high.

\section{Conclusions}

This study has been one of the first attempts to find out the application condition for the medium and high fidelity models in nonlinear static aeroelastic analysis. The capability of an iterative method in calculating the deformation of forward-swept wing is investigated by comparing experimental data and simulation results. In the given experimental arrangements, the simulation results have an acceptable accuracy compared with the wind tunnel test. The results show that the proposed method is suitable for the static aeroelastic analysis of the flexible wing undergoing large deformation with high computation efficiency.

The conclusions are summarized as follows:

(1) Although it may underestimate the displacements when large deformation occurs, it is advisable to use the proposed method in the framework of preliminary design and optimization when the computation time is concerned.

(2) For large wing deformation, the high fidelity model generates more accurate results compared to the medium fidelity model; however, its capability is limited by being time-consuming. The high fidelity model is recommended to be used in the detailed design phase, in which the accuracy of the result is more important than the computation time.

\section{Data Availability}

The numerical and experimental data used to support the findings of this study are available from the corresponding author upon request.

\section{Conflicts of Interest}

The authors declare that they have no conflicts of interest.

\section{Acknowledgments}

This work was supported by the National Natural Science Foundation of China (Grants nos. 11472216 and 11672240).

\section{References}

[1] J. R. Wright and J. E. Cooper, Introduction to Aircraft Aeroelasticity and Loads, American Institute of Aeronautics and Astronautics, Reston, VA, USA, 2nd edition, 2007.

[2] Z.-Q. Lu, D.-H. Gu, H. Ding, W. Lacarbonara, and L.-Q. Chen, "Nonlinear vibration isolation via a circular ring," Mechanical Systems and Signal Processing, vol. 136, Article ID 106490, 2020.

[3] Z.-Q. Lu, D. Wu, H. Ding, and L.-Q. Chen, "Vibration isolation and energy harvesting integrated in a stewart platform with high static and low dynamic stiffness," Applied Mathematical Modelling, vol. 89, pp. 249-267, 2021.

[4] N. Nguyen, E. Ting, and D. Chaparro, "Nonlinear large deflection theory with modified aeroelastic lifting line aerodynamics for a high aspect ratio flexible wing," in Proceedings of the 35th AIAA Applied Aerodynamics Conference, Denver, CO, USA, 2017.

[5] C. Zhang, Z. Zhou, X. Zhu, and P. Meng, "Nonlinear static aeroelastic and trim analysis of highly flexible joined-wing aircraft," AIAA Journal, vol. 56, no. 12, pp. 4988-4999, 2018.

[6] F. Afonso, J. Vale, É. Oliveira, F. Lau, and A. Suleman, "A review on non-linear aeroelasticity of high aspect-ratio wings," Progress in Aerospace Sciences, vol. 89, pp. 40-57, 2017.

[7] X. Liu, R. G. Cook, J. E. Cooper, and Q. Sun, "Static aeroelastic characteristics of a wing including geometric nonlinearities," in Proceedings of the AIAA Scitech 2019 Forum, San Diego, CA, USA, 2019.

[8] D. Tang and E. H. Dowell, "Experimental and theoretical study on aeroelastic response of high-aspect-ratio wings," AIAA Journal, vol. 39, pp. 1430-1441, 2001.

[9] G. Frulla, E. Cestino, and P. Marzocca, "Critical behaviour of slender wing configurations," Proceedings of the Institution of Mechanical Engineers, Part G: Journal of Aerospace Engineering, vol. 224, no. 5, pp. 587-600, 2010.

[10] G. Thwapiah and L. F. Campanile, "Nonlinear aeroelastic behavior of compliant airfoils," Smart Materials and Structures, vol. 19, no. 3, Article ID 035020, 2010.

[11] M. Castellani, J. E. Cooper, and Y. Lemmens, "Nonlinear static aeroelasticity of high-aspect-ratio-wing aircraft by finite element and multibody methods," Journal of Aircraft, vol. 54, no. 2, pp. 548-560, 2017. 
[12] M. J. Patil, D. H. Hodges, and C. E. S. Cesnik, "Nonlinear aeroelasticity and flight dynamics of high-altitude long-endurance aircraft," Journal of Aircraft, vol. 38, no. 1, pp. 88-94, 2001.

[13] J. Xiang, Y. Yan, and D. Li, "Recent advance in nonlinear aeroelastic analysis and control of the aircraft," Chinese Journal of Aeronautics, vol. 27, no. 1, pp. 12-22, 2014.

[14] M. Ritter, J. Dillinger, and Y. M. Meddaikar, "Static and dynamic aeroelastic validation of a flexible forward swept composite wing," in Proceedings of the 58th AIAA/ASCE/ AHS/ASC Structures, Structural Dynamics, and Materials Conference, Grapevine, TX, USA, 2017.

[15] C. Xie, L. Wang, C. Yang, and Y. Liu, "Static aeroelastic analysis of very flexible wings based on non-planar vortex lattice method," Chinese Journal of Aeronautics, vol. 26, no. 3, pp. 514-521, 2013.

[16] S. Guo, D. Li, and Y. Liu, "Multi-objective optimization of a composite wing subject to strength and aeroelastic constraints," Proceedings of the Institution of Mechanical Engineers, Part G: Journal of Aerospace Engineering, vol. 226, no. 9, pp. 1095-1106, 2012.

[17] C. Xie, Y. Meng, F. Wang, and Z. Wan, "Aeroelastic optimization design for high-aspect-ratio wings with large deformation," Shock and Vibration, vol. 2017, Article ID 2564314, 16 pages, 2017.

[18] M. Smith, M. Patil, and D. Hodges, "CFD-based analysis of nonlinear aeroelastic behavior of high-aspect ratio wings," in Proceedings of the 19th AIAA Applied Aerodynamics Conference, Anaheim, CA, USA, 2001.

[19] H. H. Mian, G. Wang, and Z.-Y. Ye, "Numerical investigation of structural geometric nonlinearity effect in high-aspect-ratio wing using CFD/CSD coupled approach," Journal of Fluids and Structures, vol. 49, pp. 186-201, 2014.

[20] H. Dang, Z. Yang, and Y. Li, "Accelerated loosely-coupled CFD/CSD method for nonlinear static aeroelasticity analysis," Aerospace Science and Technology, vol. 14, no. 4, pp. 250-258, 2010.

[21] C. Xie, Y. Liu, C. Yang, and J. Cooper, "Geometrically nonlinear aeroelastic stability analysis and wind tunnel test validation of a very flexible wing," Shock and Vibration, vol. 2016, Article ID 5090719, 17 pages, 2016.

[22] G. Y. Thwapiah and L. F. Campanile, "Experimental and numerical investigations on nonlinear aeroelasticity of forward-swept, compliant wings," Journal of Mechanical Design, vol. 134, Article ID 011009, 2012.

[23] X. Rongrong, Y. Zhengyin, Y. Kun, and W. Gang, "Composite material structure optimization design and aeroelastic analysis on forward swept wing," Proceedings of the Institution of Mechanical Engineers Part G Journal of Aerospace Engineering, vol. 233, no. 13, 2019.

[24] C. Zhang, H. Guo, B. Lv, J. Zha, and L. Yu, "Transonic static aeroelastic numerical analysis of flexible complex configuration wing," Shock and Vibration, vol. 2021, Article ID 5553304, 9 pages, 2021.

[25] R. H. Ricketts and V. R. Doggett, Wind-Tunnel Experiments on Divergence of Forward-Swept Wings, NASA, Washington, DC, USA, 1980.

[26] M. Blair and T. A. Weisshaar, "Swept composite wing aeroelastic divergence experiments," Journal of Aircraft, vol. 19, no. 11, pp. 1019-1024, 1982.

[27] S. R. Cole, "Divergence study of a high-aspect-ratio, forward swept wing," Journal of Aircraft, vol. 25, no. 5, pp. 478-480, 1988.
[28] Z. Wan, C. Yang, and C. Zou, "Divergence experiment prediction techniques of flat-plate forward-swept wing in wind tunnel," in Proceedings of the 44th AIAA/ASME/ASCE/ AHS/ASC Structures, Structural Dynamics, and Materials Conference, Norfolk, VA, USA, 2003.

[29] T. Liu, L. N. Cattafesta III, R. H. Radeztsky, and A. W. Burner, "Photogrammetry applied to wind-tunnel testing," AIAA Journal, vol. 38, pp. 964-971, 2000.

[30] T. Liu, A. W. Burner, T. W. Jones, and D. A. Barrows, "Photogrammetric techniques for aerospace applications," Progress in Aerospace Sciences, vol. 54, pp. 1-58, 2012.

[31] C. Spain, J. Heeg, T. Ivanco et al., "Assessing videogrammetry for static aeroelastic testing of a wind-tunnel model," in Proceedings of the 45th AIAA/ASME/ASCE/AHS/ASC Structures, Structural Dynamics \& Materials Conference, Palm Springs, CA, USA, 2004.

[32] T. L. Hedrick, "Software techniques for two- and three-dimensional kinematic measurements of biological and biomimetic systems," Bioinspiration \& Biomimetics, vol. 3, no. 3, Article ID 034001, 2008.

[33] T. Q. Truong, V. H. Phan, H. C. Park, and J. H. Ko, "Effect of wing twisting on aerodynamic performance of flapping wing system," AIAA Journal, vol. 51, no. 7, pp. 1612-1620, 2013.

[34] E. C. E. Culler, C. Fagley, J. Seidel, T. E. Mclaughlin, and J. A. N. Farnsworth, "Developing a reduced order model from structural kinematic measurements of a flexible finite span wing in stall flutter," Journal of Fluids and Structures, vol. 71, pp. 56-69, 2017.

[35] E. H. Dowell, "A modern course in aeroelasticity," Meccanica, vol. 34, no. 2, pp. 140-141, 1999.

[36] W. P. Rodden and E. H. Johnson, MSC/NASTRAN Aeroelastic Analysis: User's Guide, MacNeal-Schwendler Corporation, Newport Beach, CA, USA, 1994.

[37] C. D. Wieseman, "Methodology for matching experimental and analytical aerodynamic data," in Proceedings of the 29th Structures, Structural Dynamics and Materials Conference, Williamsburg, VA, USA, 1988. 